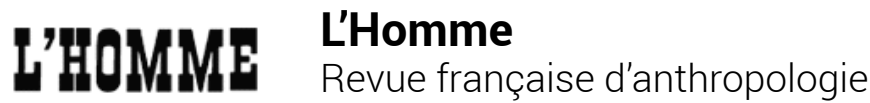

234-235 | 2020

Varia

\section{Pierre Charbonnier, Abondance et liberté. Une histoire environnementale des idées politiques}

Bernard Formoso

\section{(2) OpenEdition \\ 1 Journals}

Édition électronique

URL : https://journals.openedition.org/lhomme/37777

DOI : 10.4000//homme.37777

ISSN : 1953-8103

Éditeur

Éditions de l'EHESS

\section{Édition imprimée}

Date de publication : 14 octobre 2020

Pagination : 338-340

ISBN : 978-2-7132-2838-4

ISSN : 0439-4216

Référence électronique

Bernard Formoso, «Pierre Charbonnier, Abondance et liberté. Une histoire environnementale des idées politiques », L'Homme [En ligne], 234-235 | 2020, mis en ligne le 14 octobre 2020, consulté le 04 janvier 2023. URL : http://journals.openedition.org//homme/37777 ; DOI : https://doi.org/10.4000/lhomme 37777

Tous droits réservés 
Pierre Charbonnier

Abondance et liberté. Une histoire environnementale des idées politiques

ANS CE livre, Pierre Charbonnier conduit une enquête très approfondie sur la manière dont, depuis le XVIII ${ }^{\mathrm{e}}$ siècle, les sciences humaines et sociales ont articulé les enjeux environnementaux aux idéaux d'autonomie et d'abondance. L'auteur conçoit son histoire environnementale des idées politiques comme un point de vue qui cherche à saisir les rapports que les grandes doctrines politiques occidentales ont établis avec les formes de subsistance, les modes d'habitat (couplant territorialité et sécurité) et les sciences empiriques ayant pour objet la terre et ses ressources. Cette mise en perspective, inédite, fait ressortir des moments critiques et des controverses autour des enjeux de propriété, de production, de justice sociale, de gestion des déchets, de dérèglement climatique et de risques.

L'idéal d'autonomie, applicable à l'individu et au collectif, serait un héritage des Lumières. Il condenserait en lui les exigences d'égalité, de liberté et de propriété portées par ce courant philosophique. Jusqu'à récemment, observe l'auteur, le lien indirect qui existe entre la conquête de l'autonomie et l'exploitation des ressources planétaires était négligé par les grands paradigmes de l'économie politique, bien qu'ils corrèlent abondance et émancipation, croissance et démocratie. Selon lui, un tel aveuglement résulte de deux focales disjointes: d'une part, une histoire de la modernité rapportée à ses évolutions techniques et matérielles; de l'autre, une approche exclusivement posée dans les termes de la construction institutionnelle. Certes, le concept d'anthropocène reflète désormais une meilleure réflexivité des sociétés post-industrielles concernant l'impact environnemental de leurs activités extractives, mais cette prise de conscience ne débouche pas sur un changement de cap sociétal qui, tout en répondant à l'idéal d'autonomie, permettrait de faire face efficacement à la détérioration avancée de la biosphère. Dans ce contexte, l'objet de l'enquête de l'auteur est double: procéder à une rétrospection analytique des théories occidentales du progrès afin d'isoler leurs impasses épistémiques et les occultations idéologiques qu'elles révèlent dans leur prise en compte des limites environnementales; identifier, sur un mode prospectif, les sujets et les modes d'action qui pourraient surmonter ces obstacles et relever le défi démocratique à l'âge de la crise climatique.

Le livre, organisé en onze chapitres, isole trois «blocs historiques»: 1) la modernité préindustrielle durant laquelle la terre a servi de socle à la légitimité du pouvoir politique et son travail constitue la base de la subsistance (XVII ${ }^{\mathrm{e}}$ et XVIII ${ }^{\mathrm{e}}$ siècles); 2) la modernité industrielle amorcée au $\mathrm{XIX}^{\mathrm{e}}$ siècle, qui se façonne autour de l'usage des énergies fossiles (charbon puis pétrole) et qui aboutit à une refonte en profondeur des activités productives; 3) la mutation écopolitique entamée à partir des années 1980, qui tient compte de "l'altération catastrophique et irréversible des conditions écologiques globales» (p. 9). Chacune de ces séquences, informée par l'analyse minutieuse des textes de penseurs représentatifs, se signale par des " affordances" différentes, c'est-à-dire des prises différenciées qu'offrent la terre et, par extension, les moyens matériels d'existence à l'action politique et juridique.

$\mathrm{Au} \mathrm{XVII}{ }^{\mathrm{e}}$ siècle, les affordances politiques de la terre focalisent l'attention de philosophes comme Grotius, Hobbes ou Locke, dans le contexte des conquêtes coloniales et de l'occupation de nouveaux espaces. Le cadre juridico-politique qu'il convient de donner à l'appropriation du sol est au cœur des débats, via le traitement des questions de propriété foncière, de territorialité et les controverses autour des communs. Puis, au siècle suivant s'élabore sous l'impulsion des philosophes des Lumières un progressisme émancipateur qui articule liberté et croissance. L'effort d'éman- 
cipation est alors dirigé contre l'arbitraire politique et contre une nature jugée adverse, qu'il s'agit de dominer par l'optimisation des techniques agraires. C'est aussi à cette époque que prennent formes les premières doctrines économiques. D'un côté, explique l'auteur, la physiocratie «entend faire fructifier les fruits de la nature à travers une physiologie gouvernementale efficace» (p. 97), de l'autre, Adam Smith, à la suite de David Hume, théorise le pacte libéral en prônant une croissance intensive déliée des limites géo-écologiques, car reposant principalement sur les circulations marchandes.

$\mathrm{Au} \mathrm{XIX}^{\mathrm{e}}$ siècle, les premiers recours à l'énergie fossile entraînent une transformation en profondeur du système productif. L'autonomie est renvoyée par des penseurs comme Alexis de Tocqueville et Stanley Jevons à la capacité extractive, tandis que les limites sociétales de son expression individuelle sont mises en évidence par François Guizot. L'auteur montre que les penseurs socialistes et les premiers sociologues théorisent, pour leur part, un conflit d'orientation entre abondance et liberté. Pierre-Joseph Proudhon et Émile Durkheim, notamment, opposent au projet de l'autonomie-extraction celui de l'autonomie-intégration, en faisant de la rareté le corrélat de la compétition pour le captage des richesses, mais aussi, chez Durkheim, en pointant les dégâts moraux, psychologiques et sociaux qu'engendre la course au profit. L'industrie n'en est pas moins un facteur clé d'intégration et de stabilisation sociale pour Durkheim, Saint-Simon ou Thornstein Veblen. Chez ce dernier, la technocratie qu'incarnent les ingénieurs est même érigée en levier essentiel de la gouvernance démocratique.

Karl Marx interprète quant à lui la rupture entre croissance et émancipation sous l'angle des formes d'exploitation que le système capitaliste met en œuvre. Du fait de la valeur socialisatrice centrale qu'il confère à la production dans sa capacité à transformer les conditions matérielles d'existence, Marx est l'auteur du XIX ${ }^{e}$ siècle qui intègre le plus clairement l'écologie à sa réflexion. Pierre Charbonnier indique qu'il a mis en paral- lèle l'extorsion du travail et le pillage du sol opérés par le capitalisme. Il a aussi anticipé le risque d'épuisement des ressources auquel l'humanité est aujourd'hui confrontée. Enfin, ses analyses de l'appareil productif des sociétés industrielles de la seconde moitié du XIX ${ }^{\mathrm{e}}$ siècle révèlent leur transition des affordances du sol vers celles de la machine. Marx, cependant, réduit la terre à sa fonction économique, ce qui n'est pas le cas de Karl Polanyi, un précurseur de la réflexivité environnementale selon Pierre Charbonnier. En effet, Polanyi pense que la terre doit être exclue du marché autorégulateur, car elle ne peut être réduite à une marchandise du fait de sa fonction de moyen de subsistance primaire dont il faut protéger le potentiel, mais aussi parce qu'en tant que support d'habitat, elle joue un rôle central dans la cohésion sociale et la construction des identités. Cela dit, relève l'auteur, "le rendez-vous entre la pensée socialiste et la question de la nature a été largement manqué» (p. 284); chez Marx, parce qu'il prête à la paysannerie une absence de conscience collective, et chez Polanyi parce qu'il l'appréhende comme une force conservatrice.

Poursuivant l'examen rétrospectif de la triangulation autonomie - abondance écologie dans la pensée occidentale, Pierre Charbonnier relève une éclipse de la question environnementale au cours des Trente Glorieuses. En effet, au lendemain de la Seconde Guerre mondiale, le capitalisme démocratique devient l'horizon dominant en Occident, dans un contexte où la dynamique de reconstruction est prioritaire. La philosophie politique se détourne alors de ce que l'auteur appelle «le vieux choc métabolique de la première industrialisation " pour porter sa réflexion sur les droits de l'homme et les alternatives au consumérisme ambiant (p. 291). Certes, dans les années 1960, le mouvement de la contre-culture, incarné notamment par Herbert Marcuse, rouvrira le dossier des liens à établir entre autonomie et abondance, mais sans réelle prise en compte des affordances négatives alors en plein essor, qui réduisent la réflexivité matérielle et externalisent les coûts écologiques (projetés ailleurs ou dans le futur). 
Il faut attendre les années 1970 et la publication, par le Club de Rome, de The Limits to Growth (1972), pour que plusieurs auteurs contestent la soutenabilité du modèle de croissance linéaire hérité de la première modernité industrielle, en pointant ses limites et les risques écologiques qu'engendre son extension planétaire. Pierre Charbonnier examine entre autres les apports en ce sens d'Ulrich Beck, Anthony Giddens et Nicholas Georgescu-Roegen ${ }^{1}$. Pour Beck, la phase actuelle de la modernité correspondrait à une prise de conscience des risques générés par la moindre de nos activités et conduirait à un niveau élevé de réflexivité concernant leurs effets sur notre santé, notre environnement et notre devenir. De ce fait, la nature ne peut plus être traitée en réservoir de richesses extérieur à l'économie. Nicholas Georgescu-Roegen avait auparavant théorisé ce constat en proposant une approche bio-économique qui, prenant appui sur le principe d'entropie, redéfinissait l'économie comme l'art d'optimiser le bilan thermodynamique de la société afin d'assurer son entretien. Quant à Anthony Giddens, il dégage des horizons plus positifs au paradigme du risque, en y voyant un levier de responsabilisation et d'émancipation des citoyens de l'âge réflexif.

Les derniers développements au sein des sciences sociales que prend en compte l'étude ont trait aux propositions de Bruno Latour concernant un "parlement des choses", ainsi qu'aux théories de la symétrisation. Pour Pierre Charbonnier, l'utopie latourienne relève d'un «socialisme de la preuve» dont l'enjeu est l'acquisition d'une responsabilité épistémique de la part des citoyens, qui leur permettrait de s'imposer en contre-pouvoir efficace face au scientisme industrialiste. Il s'agit d'une utopie, car elle minimise le déséquilibre des rapports de force avec les acteurs de l'industrie. Pour sa part, le mouvement de symétrisation recouvre un ensemble hétéroclite de travaux relevant des études féministes, des subaltern et postcolonial studies, de l'histoire environnementale, ou du tournant ontologique (Philippe Descola, Tim Ingold). Leur point commun est de repenser en profondeur l'autonomisation, en opérant un « renversement gravitationnel des savoirs", qui pourrait déboucher, à terme, sur un rééquilibrage des pouvoirs en faveur des femmes, des anciens colonisés et de la nature.

La question qui reste en suspens au terme de cette reconstitution est celle de l'identité des acteurs qui pourraient porter un nouveau projet sociétal découplant autonomie et abondance, à une époque où l'on observe une exacerbation des conservatismes, ainsi qu'un renforcement de l'alliance entre forces du marché et nativisme identitaire. Le salut, selon l'auteur, pourrait provenir d'un mouvement socialiste qui aurait profondément remanié ses catégories de pensée en faveur de la symétrisation et substituerait au productivisme une forme de décroissance raisonnée, privilégiant la balance écologique.

La réflexion philosophique de Pierre Charbonnier est riche et sa réinterprétation de certains grands textes, sous l'angle des idéaux occidentaux du progrès tels qu'ils se heurtent à la finitude de la nature, apporte des éclairages très stimulants sur le processus historique de cette confrontation et les perspectives écopolitiques qui s'en dégagent. Les anthropologues regretteront sans doute le caractère très européocentré de cette analyse historique. En effet, la mise en adéquation des idéaux de prospérité et d'autonomie n'est pas l'apanage de la «modernité ». Il s'agit d'une aspiration commune à la plupart des sociétés humaines, mais selon des modalités de mise en œuvre très différentes de l'Occident et qui s'avèrent souvent plus respectueuse de l'environnement. Elles auraient donc pu offrir une source d'inspiration supplémentaire à l'auteur dans sa quête de formules écopolitiques susceptibles de relever les défis contemporains.

\section{Bernard Formoso}

1. Cf. : Ulrich Beck, La Société du risque. Trad. de l'allemand par Laure Bernardi. Paris, Flammarion, 2008 ("Champs. Essais»); Anthony Giddens, «Risk and Responsability", The Modern Law Review, 1999, 62 (1) : 1-10; Nicholas Georgescu-Roegen, The Entropy Law and the Economic Process, Cambridge, Harvard University Press, 1971. 